\title{
Diabetes Mellitus in a Population of 180,000 Insured Dogs: Incidence, Survival, and Breed Distribution
}

\author{
Tove Fall, Helene Hansson Hamlin, Åke Hedhammar, Olle Kämpe, and Agneta Egenvall
}

\begin{abstract}
Background: Canine diabetes mellitus (DM) is a common endocrinopathy with an unclear etiology. For a better understanding of the underlying mechanisms, there is a need for comprehensive epidemiologic studies. Earlier studies have shown that the risk of disease is higher in certain dog breeds.

Hypothesis: Incidence, age of onset, survival and sex proportion of DM vary by breed.

Animals: Data from a cohort of 182,087 insured dogs aged 5-12 years accounting for 652,898 dog-years at risk were studied retrospectively.

Methods: Incidence rates by sex, breed, and geography were calculated with exact denominators. Age-specific incidence and survival after 1st DM claim were computed with Cox's regression and Kaplan-Meier survival function. Multivariable survival analysis was performed for the outcome diagnosis of DM with age, sex, and geography tested as fixed effects, previous endocrine or pancreatic diseases tested as time-dependent covariates, and breed tested as a random effect.

Results: The mean age at 1st insurance claim for the $860 \mathrm{DM}$ dogs $(72 \%$ females) was 8.6 years. The incidence of DM was 13 cases per 10,000 dog-years at risk. Australian Terriers, Samoyeds, Swedish Elkhounds, and Swedish Lapphunds were found to have the highest incidence. The proportion of females with DM varied significantly among breeds. Swedish Elkhounds, Beagles, Norwegian Elkhounds, and Border Collies that developed DM were almost exclusively females. The multivariable model showed that breed, previous hyperadrenocorticism, and female sex were risk factors for developing DM. Median survival time was 57 days after 1st claim. Excluding the 223 dogs that died within 1 day, the median survival time was 2 years after 1st claim of DM.

Conclusion: The significant breed-specific sex and age differences shown in this study indicate that genetic variation could make breeds more or less susceptible to different types of DM.
\end{abstract}

Key words: Age; Endocrinology; Epidemiology; Frailty; Sex.

D iabetes mellitus (DM) is a common endocrinopathy in dogs, with certain breeds shown to have either an increased or decreased risk of developing the disease. ${ }^{1-6}$ The etiology of canine DM is considered multifactorial and may be broadly divided into insulin resistance and insulin deficiency. ${ }^{7}$ Insulin deficiency diabetes is suggested to be caused either by autoimmune destruction of insulinproducing beta cells, pancreatitis, or secondary to chronic hyperglycemia (eg, insulin resistance diabetes) ${ }^{7-10}$ Insulin resistance diabetes may occur as a consequence of hormonal disturbances (eg, hyperadrenocorticism, progesterone-induced acromegaly). ${ }^{11,12}$ Epidemiologic studies have reported that most affected dogs are $>5$ years of age at the onset of DM. ${ }^{2,3}$ Several studies have found female dogs to have an increased risk for $\mathrm{DM}^{2,6}$; however, in 1 recent large study this association was not confirmed. ${ }^{3}$ Dogs with DM are most often dependent on insulin for survival. The mean survival time in dogs with DM is described to be approximately 3 years from the time of diagnosis with the highest mortality occurring during the 1 st 6 months. ${ }^{13}$ One study reported that $64 \%$ of DM dogs that survive initial stabilization also survive the 1st year after diagnosis. ${ }^{14}$ Studies have shown different risk estimates for human DM in different nations, seasons of the year, and in rural versus urban areas..$^{15-17}$ Some

From the Department of Clinical Sciences, Faculty of Veterinary Medicine and Animal Science, Swedish University of Agricultural Sciences, SE-750 07 Uppsala, Sweden (Fall, Hamlin, Hedhammar, Egenvall); and the Department of Medical Sciences, University Hospital, Uppsala University, SE-751 85 Uppsala, Sweden (Kämpe). Reprint requests: Tove Fall, Department of Clinical Sciences, Box 7054, SE-750 07 Uppsala, Sweden; e-mail: tove.fall@kv.slu.se.

Submitted January 24, 2007; Revised April 10, 2007; Accepted May 15, 2007.

Copyright $(02007$ by the American College of Veterinary Internal Medicine

0891-6640/07/2106-0008/\$3.00/0 studies of dogs have indicated a winter peak for the onset of DM, ${ }^{3,18}$ whereas other studies have shown no seasonal predisposition., ${ }^{2,6}$

A large proportion of the Swedish dog population is covered by an insurance plan, and most dogs are not neutered. The Agria Insurance Company ${ }^{\mathrm{a}}$ covers approximately $30 \%$ of the entire Swedish dog population. ${ }^{19}$ The insured population reflects the general Swedish dog population with regard to sex, age, and breed, with exception of mixed breed dogs and older dogs, which are underrepresented in the insured population compared with in the general Swedish dog population. ${ }^{19}$

The main objective of this study was to provide population-based breed-specific information on canine DM in dogs $>5$ years of age, regarding general incidence, incidence by age and sex, and survival after diagnosis of DM. A 2nd objective was to evaluate sex, geography, and other endocrine or pancreatic diseases as risk factors for the development of DM, controlling for age and breed.

\section{Material and Methods}

\section{Insurance Process}

The Agria Insurance Company offers 2 kinds of insurance plans for dogs. One is for veterinary care in which the owner, in the event of disease, is reimbursed for costs exceeding the deductible for veterinary treatment. The other is a life insurance plan in which the owner is reimbursed the monetary value of the dog in case of death caused by disease or accident. The veterinary care plan currently has no age limit, whereas the life insurance plan has an upper age limit of 10 years. The insurance process has been described in detail by Egenvall et al. ${ }^{20}$ Most dogs are enrolled in insurance as puppies, but dogs can enter the insurance program until they are 6 years of age. ${ }^{21}$

We used individual data on sex, breed, date of birth, date of death, date of entry and exit from insurance plan, reason for exit, date of insurance claim, diagnostic coding, postal code of the owner, and type of insurance. If more than 1 diagnosis is reported 
on the same claim, only 1 diagnostic code is recorded in the database. Neuter status was not available in the database.

\section{Data Management}

Breeds were classified according to the Swedish Kennel Club breed classification system. Some breeds were combined (eg, German Pointer included both smooth-haired and wire-haired German Pointers).

The owners' postal codes were used to construct the variable geography (south, middle, north) and whether the dog lived in 1 of the 3 largest cities in Sweden or not (urban or rural). These 2 variables were combined into 1 variable (eg, south, urban). Because none of the 3 cities are situated in the north region, there were 5 different geographic categories.

Diagnostic codes were assigned by the attending veterinarian based on a standardized system with approximately 8,000 codes. ${ }^{22}$ There were 4 codes associated with diabetes mellitus: DM, DM with complications, DM without complications, and DM with ketoacidosis. For the variable "other endocrine or pancreatic disease," the following coded diagnoses were used: hypothyroidism, hyperadrenocorticism (including iatrogenic hyperadrenocorticism), adrenocortical insufficiency, pancreatitis (acute or chronic), and exocrine pancreatic insufficiency.

\section{Study Population}

Dogs that were covered by an insurance plan for both veterinary care and life during the years 1995-2004 were selected. An individual was considered a case of DM at the time of the 1st recorded claim. Because few dogs develop DM before 5 years of age, ${ }^{2,3}$ we decided to exclude both cases $(\mathrm{n}=36)$ and time-at-risk before 5 years of age. Dogs were followed up to 10 years of age for life insurance claims and up to 12 years of age for veterinary care claims. Data after 12 years of age were considered unstable due to the low number of individuals at risk, and the cases of DM $(\mathrm{n}=19)$ $>12$ years of age at 1 st claim were excluded. Consequently, we increased the number of dogs that were followed for the same age span (from 5 to 12 years of age). The variables "other endocrine or pancreatic disease" were recorded in the same manner. Dogs ( $\mathrm{n}=$ 300 ) without match to the geographic variable were omitted.

\section{Univariable Statistics}

Descriptive statistics were presented for the study population and for the DM cases. The proportion of females and the mean age at 1 st claim with $95 \%$ confidence intervals (CI) were calculated in general and for breeds with at least 15 cases. Only data on breeds with $>4,000$ dog years at risk (DYAR) or $>10$ cases of DM were used in the breed-specific analysis. The proportion of new cases in the time periods January-March, April-June, July-September, and October-December were calculated separately for each sex with $95 \%$ CI. For cases, breed-specific proportions of females and by season and sex for the 1st claim, exact binomial 95\% CIs were constructed. Nonoverlapping CIs for sex, age, and season were considered statistically significantly different.

Incidence rates were calculated with exact denominators. Each animal in the study population contributed to the denominator with the exact time they were at risk in the database. Incidence rates were calculated in general, by the 1990-1992 cohorts, breed, sex, and geography. The incidence rates were multiplied by 10,000 to be interpreted as the number of new cases of DM per 10,000 DYAR. Cases of other endocrine or pancreatic diseases were counted in the non-DM population and before and after onset of $\mathrm{DM}$ in the DM population.

By using the baseline survival statement from Cox's regression, the cumulative proportions of dogs that developed DM up to certain ages (ie, 8, 10, and 12 years) were estimated. The proportions also were estimated by breed for breeds with $\geq 40$ cases. For the analysis, we assumed that the risk of DM before 5 years of age was 0 . Age-specific and breed- and age-specific incidence rates were constructed by use of the SMOOTH macro, ${ }^{23}$ which computes age-specific hazards (rates) from the baseline survival function computed by PHREG. ${ }^{\mathrm{b}}$ This approach provides a smoothed estimate of the hazard curve by a kernel-smoothing method. The WIDTH parameter was set to one fifth of the range of event times.

Kaplan-Meier survivor functions (up to 10 years of age) for survival after 1st DM claim were constructed for all dogs and by breed for breeds with at least 40 cases. The log rank test was used to evaluate overall, and if significant, pairwise differences in survival among these breeds. Survival times also were estimated for dogs surviving $>1$ day and $>30$ days after 1 st DM claim.

\section{Multivariable Analysis of Time to DM}

A multivariable Cox regression model was developed for a reduced dataset consisting of the breeds with $>4,000$ DYAR and the breeds with at least 10 cases of DM. The outcome was time to 1 st DM event. Dogs were censored either when the study period ended, when they reached 12 years of age, or when they left the insurance program for reasons unrelated to DM. Sex and geography were entered as fixed effects (male baseline for sex and middle-rural baseline for geography). The proportional hazards assumption was investigated by plotting the natural logarithm of the cumulative hazard stratified by sex and geography (log-log plots; from Cox regressions without covariates) against the $\log$ of age of exit. A frailty term (random effects in survival analysis) was entered for breed. The 1st model (i) was constructed using only these non-time-dependent effects.

In order to investigate the "other endocrine or pancreatic" diseases as possible risk factors for the development of DM, each of these was tested one at a time, together with the fixed effects from model (i) resulting in 5 interim models. If the time-dependent covariates were significant at $P<.1$, they were further tested in models (ii)-(vi) together with both the fixed and frailty effects from model (i). For each modeled "other endocrine or pancreatic" disease, the dataset was split at the time of 1 st claim for this disease, and the effect was calculated to be the same from this event until $\mathrm{DM}$ or censoring.

Model fit was inspected by use of plots of Martingale residuals against DYAR and against covariates.

\section{Multivariable Analysis of Time to Death after DM}

Multivariable Cox regression analysis was further employed to evaluate risk factors for survival after the 1st claim of DM. Only time up to 10 years of age was used. The possible fixed risk factors tested were age at DM, sex, geography, and previous diagnosis (before 1st DM claim) of hyperadrenocorticism, adrenocortical insufficiency, or pancreatitis. Breed was entered as a frailty effect, a priori with the 46 breeds from model (i). Consequently, only breeds with at least 2 individuals were kept and 3 breeds (with 1 individual each) were deleted. Interactions were tested at $P<.1$, and effects were retained if $P<.05$. Model validation was made analogously to the "time to DM" models. The statistical software program STATA $9.1^{\mathrm{c}}$ was used for multivariable analysis, the procedure STCOX was used for Cox regression. Data handling was performed by SAS version 9.1. ${ }^{\mathrm{b}}$

\section{Results}

\section{Study Population}

The study population included 182,087 (50.6\% female) dogs accounting for 667,282 DYAR with 294 
different breed designations recorded. The time at risk that each dog contributed ranged from 1 day to 7 years (median, 3.5 years).

\section{Cases}

Eight hundred and sixty dogs had claims for DM. The mean age at 1 st claim of DM was 8.6 years (range, 5-12 years). Of the 686 dogs that had claims for DM before 10 years of age, $252(37 \%)$ had both life and veterinary care claims, $157(23 \%)$ had life claims alone, and $277(40 \%)$ had veterinary care claims alone. Six hundred and eighteen $(72 \%)$ of the DM cases were female. The number of cases per breed with the proportion of females and the mean age of 1 st insurance claim are presented in Table 1. Cases of other endocrine or pancreatic diseases in the non-DM population and before and after onset of DM in the DM population are shown in Table 2. There was a significantly higher proportion of new female cases in the spring (AprilJune) than in the other months. No seasonality of $1 \mathrm{st}$ claim of disease in male dogs was found (Fig 1).

\section{Incidence}

Table 3 shows the incidence for all dogs, for cohorts born 1990-1992, by sex and by geographic region. Table 1 presents the breed-specific incidence rates for 46 breeds with $>4,000$ DYAR or at least 10 cases. Figure 2 shows age-specific incidence rates for breeds with $\geq 40$ cases. Table 4 presents the estimated proportion of dogs that developed DM before 8, 10, and 12 years, respectively, overall, by sex and by breed (with at least 40 cases).

\section{Survival after DM}

The median survival time $(n=686$, deaths $=413)$ after 1st DM claim was 57 days (95\% CI, 27-100). For the dogs surviving at least 1 day $(n=463$, deaths $=207)$ after diagnosis, the median survival time was 2.0 years (95\% CI, 1.1 to not estimable) and, for the dogs surviving at least 30 days $(n=347$, deaths $=105)$ after 1st DM claim, the median survival time was not estimable because of the fraction failing to reach $50 \%$ mortality (Fig 3). The proportion of dogs surviving 1 year (95\% CI) was 40\% (36-44); 2 years, 36\% (32-40); and 3 years, 33\% (29-37). The log rank test showed a significant $(P=.0013)$ difference between the estimated survival times after 1st DM claim for the 5 breeds with at least 40 cases (Fig 4). Significant differences in the pairwise comparison were found between the Border Collie and the Drever $(P=.004)$, the Border Collie and the Swedish Elkhound $(P<.001)$, the Labrador and the Swedish Elkhound $(P=.04)$, and the Samoyed and the Swedish Elkhound $(P=.02)$.

\section{Multivariable Analysis of Time to DM}

Model (i) included 139,861 dogs with 509,025 DYAR and 702 DM cases from 46 different breeds, with an incidence of 13.8 cases per 10,000 DYAR. Females had a hazard ratio (HR) of 2.6 (95\% CI, 2.2-
3.1) compared with males. The tested fixed effect geography was not significant. The frailty effect for breed was significant $(P<.001)$. Hyperadrenocorticism was added as a time-dependent covariate in model (ii). The HR for dogs with hyperadrenocorticism was 9.3 (95\% CI, 5.4-15.9) compared with dogs without hyperadrenocorticism, whereas the sex effect was the same. Model (ii) was based on 139,854 dogs (7 dogs were omitted because of different handling of decimals in the 2 programs used) with 702 DM cases. The rank of the breed-specific frailty components from model (ii) is presented in Table 1. Pancreatitis, pancreatic insufficiency, adrenocortical insufficiency, and hypothyroidism were evaluated as risk factors in models (iii)(vi), but these variables did not contribute to the models, neither increasing nor decreasing the risk of DM. The plots of Martingale residuals against DYAR and covariates were deemed acceptable. In model (i), the largest negative residuals were found for female dogs of high-risk breeds without DM. In model (ii), these were found for female dogs with hyperadrenocorticism without DM.

\section{Multivariable Analysis of Time to Death after DM}

Three hundred and sixty-five dogs (173 failures) from 39 breeds (2-32 individuals per breed) were included in the model. The breed frailty effect was significant $(P=$ .032) on survival after 1st DM claim (breed rank not shown). None of the other variables evaluated as risk factors were found to influence survival time after DM: age at 1st claim, sex, geography, or previous diagnosis (before 1st DM claim) of hyperadrenocorticism, adrenocortical insufficiency, or pancreatitis. The plots of Martingale residuals against time (in years) showed that the largest positive and smallest negative residuals were found at small values of time. Obviously, this model lacked unmeasured covariates, although breed contributed to the model.

\section{Discussion}

Published estimates of disease frequency and breed predilection of diseases in companion animals commonly are based on hospital records from teaching hospitals, which lack information from primary care units and about the size and composition of the population at risk. In the present study we followed insured dogs longitudinally with health care information from all levels of veterinary care. The authors' opinion, based on previous work, is that the results can be extrapolated to all insured dogs in Sweden. ${ }^{19}$ Stratified estimates (eg, by breed and sex) are most probably relevant for dogs in industrialized countries all over the world. The obvious exception is that Swedish dogs are not commonly neutered as in most other Western dog populations.

The overall incidence of DM in this study was 13 cases per 10,000 DYAR. For the birth cohorts 19901992, which were followed to 12 years of age, the incidence was higher (16 cases per 10,000 DYAR) because the incidence of DM increases with age. The estimated cumulative proportion of dogs that would 
Table 1. Breed-specific details of age at 1st claim, gender and incidence from an insured population of 182,087 dogs with a total of 860 diabetes mellitus cases.

\begin{tabular}{|c|c|c|c|c|c|c|c|}
\hline Breed & $\mathrm{n}$ & $\begin{array}{l}\text { Mean Age } \\
(95 \% \text { CI })\end{array}$ & $\begin{array}{l}\text { Prop Females } \\
\quad(95 \% \mathrm{CI})\end{array}$ & Rank & AR I & DYAR & $\begin{array}{l}\text { IR, cases per } 10,000 \\
\text { DYAR }(95 \% \mathrm{CI})\end{array}$ \\
\hline Australian Terrier & 10 & - & - & $1-1$ & 165 & 548 & $183(69-296)$ \\
\hline Samoyed & 40 & $8.6(8.0-9.1)$ & $0.63(0.46-0.77)$ & $2-2$ & 1,087 & 3,850 & $104(72-136)$ \\
\hline Swedish Lapphund & 13 & - & - & $3-3$ & 508 & 1,803 & $72(33-111)$ \\
\hline Swedish Elkhound & 47 & $7.8(7.4-8.3)$ & $0.96(0.86-0.99)$ & 44 & 3,485 & 10,359 & $45(32-58)$ \\
\hline Border Collie & 51 & $8.9(8.5-9.3)$ & $0.98(0.90-1.00)$ & $5-5$ & 3,999 & 13,990 & $36(26-46)$ \\
\hline Finnish Hound & 17 & $8.5(7.8-9.1)$ & $0.65(0.38-0.86)$ & $6-6$ & 1,571 & 4,746 & $36(19-53)$ \\
\hline Drever & 54 & $8.6(8.3-8.9)$ & $0.83(0.71-0.92)$ & $7-7$ & 4,580 & 15,187 & $36(26-45)$ \\
\hline West Highland White Terrier & 24 & $9.2(8.6-9.8)$ & $0.58(0.37-0.78)$ & $8-8$ & 1,778 & 7,275 & $33(20-46)$ \\
\hline Hamilton Hound & 30 & $8.1(7.5-8.7)$ & $0.67(0.47-0.83)$ & $9-9$ & 3,022 & 10,492 & $29(18-39)$ \\
\hline Beagle & 15 & $8.9(8.2-9.6)$ & $0.93(0.68-1.00)$ & $10-11$ & 1,680 & 6,200 & $24(12-36)$ \\
\hline Poodle (Miniature and Toy) & 35 & $8.5(8.0-9.1)$ & $0.66(0.48-0.81)$ & $11-12$ & 3,785 & 14,492 & $24(16-32)$ \\
\hline Rottweiler & 20 & $7.9(7.2-8.5)$ & $0.35(0.15-0.59)$ & $12-10$ & 2,877 & 8,832 & $23(13-33)$ \\
\hline Cairn Terrier & 22 & $9.3(8.8-9.8)$ & $0.68(0.45-0.86)$ & $13-13$ & 2,605 & 10,609 & $21(12-30)$ \\
\hline Miniature Schnauzer & 14 & - & - & $14-14$ & 1,896 & 7,035 & $20(10-30)$ \\
\hline Finnish Spitz & 8 & - & - & $15-15$ & 1,302 & 4,405 & $18(6-31)$ \\
\hline Bichon Frisée & 13 & - & - & $16-17$ & 1,954 & 7,507 & $17(8-27)$ \\
\hline Norwegian Elkhound & 18 & $8.4(7.8-8.9)$ & $1.00\left(0.81-1^{*}\right)$ & $17-16$ & 3,150 & 10,655 & $17(9-25)$ \\
\hline Mixed breed & 33 & $8.6(8.1-9.2)$ & $0.91(0.76-0.98)$ & $18-19$ & 6,253 & 22,083 & $15(10-20)$ \\
\hline Cavalier King Charles Spaniel & 23 & $7.8(7.3-8.4)$ & $0.52(0.31-0.73)$ & $19-18$ & 4,507 & 15,857 & $15(9-20)$ \\
\hline English Springer Spaniel & 23 & $8.3(7.6-9.1)$ & $0.87(0.66-0.97)$ & $20-22$ & 4,565 & 17,165 & $13(8-19)$ \\
\hline Labrador & 50 & $8.9(8.4-9.4)$ & $0.52(0.37-0.66)$ & $21-20$ & 9,483 & 36,715 & $13(10-17)$ \\
\hline Irish Setter & 7 & - & - & $22-21$ & 1,386 & 5,276 & $13(3-23)$ \\
\hline Tervueren & 5 & - & - & $23-23$ & 1,189 & 4,555 & $11(1-21)$ \\
\hline Shetland Sheepdog & 10 & - & - & $24-25$ & 2,665 & 9,804 & $10(4-17)$ \\
\hline Munsterlander & 4 & - & - & $25-26$ & 978 & 4,030 & $10(0-20)$ \\
\hline Dalmatian & 4 & - & - & $26-27$ & 991 & 4,080 & $10(0-19)$ \\
\hline Dachshund (smooth/wire/normal) & 27 & $9.1(8.5-9.7)$ & $0.74(0.54-0.89)$ & $27-38$ & 7,420 & 30,341 & $9(6-12)$ \\
\hline Bernese Mountain Dog & 4 & - & - & $28-24$ & 1,643 & 4,560 & $9(0-17)$ \\
\hline Border Terrier & 6 & - & - & $29-29$ & 1,928 & 7,483 & $8(2-14)$ \\
\hline English Cocker Spaniel & 8 & - & - & $30-31$ & 2,835 & 10,321 & $8(2-13)$ \\
\hline Flat-Coated Retriever & 7 & - & - & $31-30$ & 2,704 & 9,274 & $8(2-13)$ \\
\hline German Pointer & 6 & - & - & $32-32$ & 2,327 & 8,235 & $7(2-13)$ \\
\hline Yorkshire Terrier & 4 & - & - & $33-35$ & 1,500 & 5,823 & $7(0-14)$ \\
\hline Nova Scotia Duck Tolling Retriever & 3 & - & - & $34-33$ & 1,293 & 4,504 & $7(0-14)$ \\
\hline Petite Basset Griffon & 3 & - & - & $35-34$ & 1,309 & 4,566 & $7(0-14)$ \\
\hline Jack Russell Terrier & 4 & - & - & $36-36$ & 1,838 & 6,538 & $6(0-12)$ \\
\hline Shih Tzu & 3 & - & - & $37-37$ & 1,325 & 5,033 & $6(0-13)$ \\
\hline Miniature Dachshund & 12 & - & - & $38-38$ & 5,820 & 20,873 & $6(3-9)$ \\
\hline German Shepherd & 16 & $8.3(7.6-9.0)$ & $0.94(0.70-1.00)$ & $39-39$ & 13,522 & 45,456 & $4(2-5)$ \\
\hline Rough Haired Collie & 3 & - & - & $40-40$ & 3,237 & 12,271 & $2(0-5)$ \\
\hline Standard Poodle & 1 & - & - & $41-42$ & 1,643 & 5,958 & $2(0-5)$ \\
\hline Soft Coated Wheaten Terrier & 1 & - & - & $42-41$ & 1,715 & 6,104 & $2(0-5)$ \\
\hline Bearded Collie & 1 & - & - & $43-43$ & 1,866 & 7,511 & $1(0-4)$ \\
\hline Golden Retriever & 3 & - & - & $44-46$ & 11,099 & 44,758 & $0(0-1)$ \\
\hline Boxer & - & - & - & $45-44$ & 1,594 & 5,253 & 0 \\
\hline Papillon & - & - & - & $46-45$ & 1,782 & 6,615 & 0 \\
\hline
\end{tabular}

n, number of animals; prop females, proportion of females; CI, confidence interval; Rank, crude rank and rank from Cox proportional frailty model (Crude rank-Model rank); AR, animals at risk; DYAR, dog years at risk; IR, incidence rate in DM cases/10,000 DYAR.

${ }^{\text {a }}$ Proportion of females and mean age at 1 st claim are only estimated for breed with more than 15 cases.

*Normally, all confidence intervals (CI) are two-sided, i.e they show the variation for the estimate with an upper and a lower limit with a $95 \%$ confidence. The CIs in this case are calculated with exact binomial distribution, thus one-sided, $97.5 \%$ CI.

develop DM before 12 years of age was $1.2 \%$ (ie, 1 of 100 dogs reaching 12 years of age would develop DM). Earlier epidemiologic reports on DM have neither been estimated incidence nor determined cumulative proportion, which makes comparison difficult. Davison et al reported from a UK insurance cohort a DM prevalence of $0.32 \% .^{3}$ Guptill and coworkers ${ }^{2}$ found a hospital prevalence of $0.64 \%$ in the United States, and Fracassi and coworkers ${ }^{1}$ reported an Italian hospital prevalence of $1.33 \%$.

As any secondary database, an insurance database cannot be expected to have completely accurate recording of diagnoses. The Agria database has been validated with regard to the agreement between veter- 
Table 2. Distribution of 5 endocrine or pancreatic diseases of $860 \mathrm{DM}$ cases and 181,227 dogs without DM aged 5-12 years.

\begin{tabular}{lccc}
\hline & $\begin{array}{c}\text { Cases before } \\
\text { DM Diagnosis }\end{array}$ & $\begin{array}{c}\text { Cases after } \\
\text { DM Diagnosis }\end{array}$ & $\begin{array}{c}\text { Cases in } \\
\text { Non-DM } \\
\text { Dogs }\end{array}$ \\
\hline $\begin{array}{l}\text { Hypothyroidism } \\
\text { Adrenocortical }\end{array}$ & 7 & 3 & 1345 \\
$\quad$ insufficiency & 3 & 1 & 207 \\
$\begin{array}{l}\text { Hyperadrenocorticism } \\
\text { Exocrine pancreatic }\end{array}$ & 17 & 16 & 733 \\
$\quad$ insufficiency & 0 & 1 & 91 \\
Pancreatitis & 7 & 2 & 300 \\
\hline
\end{tabular}

DM, diabetes mellitus.

inary practice records and computerized insurance data. The observed agreement for diagnosis in health and life claims was 84.0 and $84.9 \%$, respectively, which was considered as fair diagnostic validity. ${ }^{24}$ We believe that the DM diagnosis has a higher diagnostic validity than many other diseases in view of the straightforward clinical presentation and readily interpreted clinical biochemistry findings.

In the present study, $72 \%$ of the DM cases were female dogs, which is consistent with several previous studies. ${ }^{2,6,14}$ The high incidence of DM in intact female dogs can be explained by the insulin antagonistic action of progesterone and mammary-derived growth hormone. ${ }^{12}$ In Sweden, spaying of bitches is rare (approximately $7 \%$ in a cross-sectional sample) and is performed most often late in life for medical reasons such as pyometra. ${ }^{19}$ Unfortunately, neuter status is not recorded in the database.

A tendency toward spring (April-June) predisposition was recorded for the female dogs in the present study. This spring tendency may reflect onset of progesterone-induced DM triggered by increased estral activity. The date of 1st DM insurance claim, however, could occur at a considerable time after the 1st signs of disease, as some dog owners do not seek help at the 1st

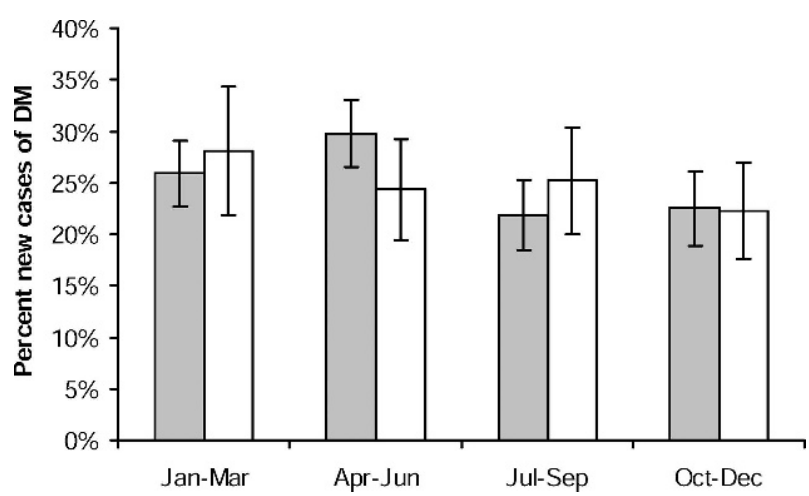

Fig 1. Seasonal distribution of date of 1st DM insurance claim in $860 \mathrm{DM}$ cases grouped by sex (female $=$ grey) in Swedish insured dogs during 1995 to 2004. Error bars represent 95\% exact binomial confidence intervals. Dogs insured by Agria Insurance Company, Stockholm, Sweden.
Table 3. Incidence rates for all dogs $(n=182,087)$ in the study, for the birth cohorts 1990-1992 and by sex and geographic region.

\begin{tabular}{lcrc}
\hline \multicolumn{1}{c}{ Animals } & $\mathrm{n}$ & DYAR & $\begin{array}{c}\text { IR, Cases per 10,000 } \\
\text { DYAR }(95 \% \text { CI })\end{array}$ \\
\hline All & 860 & 652,898 & $13(12-14)$ \\
Cohorts 1990-1992 & 483 & 296,489 & $16(15-18)$ \\
Male & 242 & 318,406 & $8(7-9)$ \\
Female & 618 & 334,491 & $19(17-20)$ \\
North rural & 118 & 74,154 & $16(13-19)$ \\
Middle rural & 311 & 214,833 & $15(13-16)$ \\
Middle urban & 108 & 87,759 & $12(10-15)$ \\
South rural & 244 & 205,835 & $12(10-13)$ \\
South urban & 79 & 70,318 & $11(9-14)$ \\
\hline
\end{tabular}

$\mathrm{n}$, number of animals; DYAR, dog years at risk; IR, incidence rate; $\mathrm{CI}$, confidence interval.

signs of disease. In 2 previous studies, a winter predisposition was found for the onset of DM in dogs. ${ }^{3,18}$ Two other studies, however, did not show any seasonal predisposition., ${ }^{2,6}$ In type $1 \mathrm{DM}$ of humans, seasonal variability in diagnosis attracted much attention in the 1970s. ${ }^{25}$ Seasonality of onset of type $1 \mathrm{DM}$ conforms to a sinusoidal model with a peak occurring in winter, a feature that is consistently observed in both sexes and in all age groups. ${ }^{26}$ Of interest, a large multicenter epidemiologic study showed that no winter peak was found in the Scandinavian countries. ${ }^{16}$ The reasons why DM shows neither a winter predisposition in the present study of dogs nor in Scandinavian people are not known.

DM is mainly a disease of middle-aged and older dogs. ${ }^{2,3,14,18}$ Canine DM thus differs from the human autoimmune type $1 \mathrm{DM}$ in humans, which is diagnosed usually in young age, although it has become more and more apparent that type $1 \mathrm{DM}$ occurs in all age groups. The late onset form of autoimmune DM is sometimes

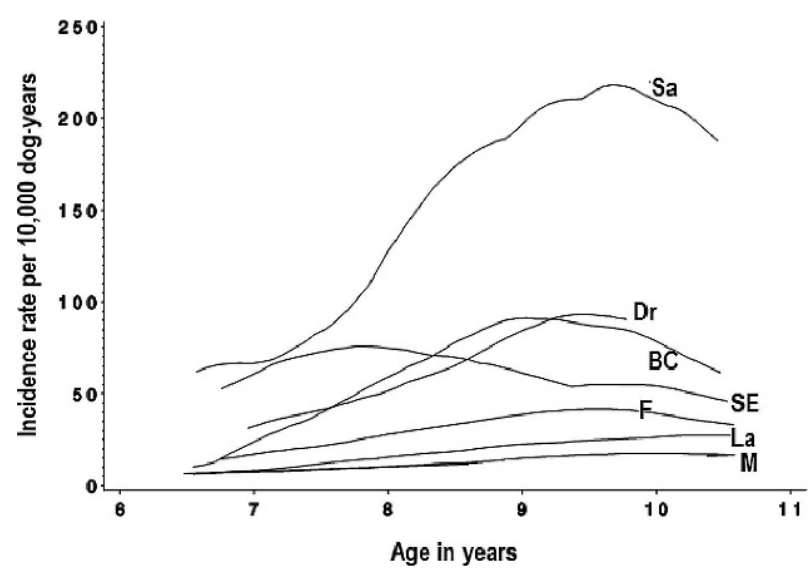

Fig 2. Smoothed estimates of age-specific incidence rates for 5 breeds with at least $40 \mathrm{DM}$ cases each and for all cases in a life and veterinary care insured population of $182,087 \mathrm{dogs}$ in Sweden during 1995 to 2004. Dogs insured by Agria Insurance Company, Stockholm, Sweden. Samoyed (Sa), Drever (Dr), Border Collie (BC), Swedish Elkhound (SE), Labrador (La), All females (F), All males (M). Dogs insured by Agria Insurance Company. 
Table 4. Cumulative percentage of dogs that have developed diabetes mellitus at different ages.

\begin{tabular}{lccc}
\hline & \multicolumn{3}{c}{ Percentage (95\% CI) } \\
\cline { 2 - 4 } & 8 Years & 10 Years & 12 Years \\
\hline All & $0.3(0.2-0.3)$ & $0.7(0.7-0.8)$ & $1.2(1.1-1.2)$ \\
Females & $0.4(0.3-0.4)$ & $1.0(0.9-1.1)$ & $1.6(1.5-1.7)$ \\
Males & $0.1(0.1-0.2)$ & $0.4(0.3-0.5)$ & $0.6(0.5-0.7)$ \\
Border Collie & $0.4(0.1-0.6)$ & $2.2(1.5-2.8)$ & $3.4(2.3-4.4)$ \\
Swedish Elkhound & $1.3(0.8-1.8)$ & $2.5(1.7-3.3)$ & $3.6(2.3-4.9)$ \\
Samoyed & $1.9(0.9-2.8)$ & $5.3(3.4-7.1)$ & $9.0(5.9-12.0)$ \\
Labrador & $0.2(0.1-0.3)$ & $0.6(0.4-0.9)$ & $1.3(0.9-1.7)$ \\
Drever & $0.7(0.4-1.0)$ & $2.4(1.7-3.1)$ & $3.0(2.2-3.9)$ \\
\hline
\end{tabular}

CI, confidence interval.

called latent autoimmune diabetes in adults (LADA). It remains unknown why canine DM is diagnosed primarily in older aged dogs. Although it is reasonable to assume that insulin-resistant types of canine DM (eg, secondary to hyperadrenocorticism or acromegaly) start late in life, this explanation does not hold for all canine DM cases. An earlier study showed that approximately $80 \%$ of dogs with DM were 5 to 12 years of age at onset, ${ }^{3}$ and another study indicated that $41 \%$ were $>11$ years of age at 1 st visit to tertiary care facilities. ${ }^{2}$ In the present study, only dogs aged 5 to 12 years were included. Therefore, conclusions could only be made for the age group studied. Because life insurance for dogs is not available after 10 years of age, the sensitivity of detection of disease in older dogs might be low (because the claim must exceed a deductible), and disease recording for veterinary claim may be less precise than for life claims.

In this study, no significant differences were found in the incidence of DM among different areas of the country, although speciality care for companion animals is more readily available in the cities. This result could

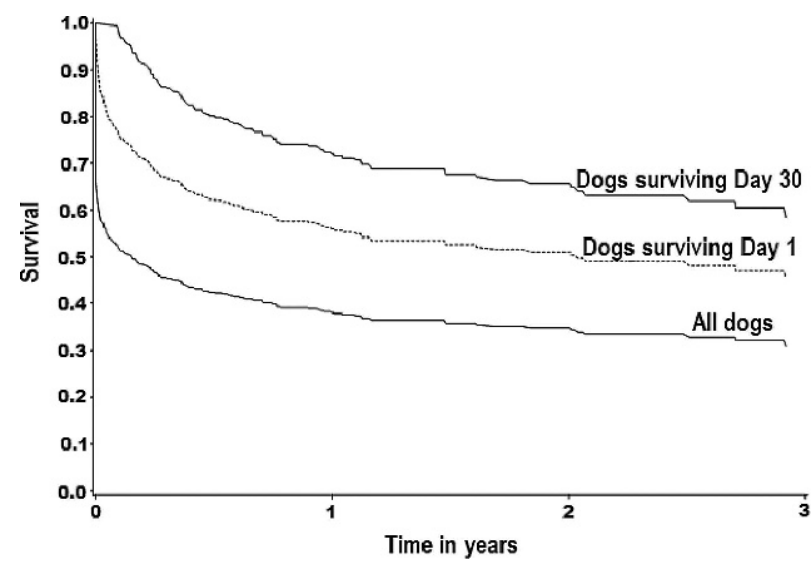

Fig 3. Kaplan-Meier survival estimates after 1st insurance claim for DM in dogs 5-10 years of age with life insurance during 1995 to 2004 in Sweden. The 1st group includes all dogs $(\mathrm{n}=686$, deaths $=$ 413). The 2nd group includes only the dogs that survived the 1st day $(n=463$, deaths $=207)$, and the 3 rd group includes only the dogs that survived the 1 st 30 days $(\mathrm{n}=347$, deaths $=105)$. Dogs insured by Agria Insurance Company, Stockholm, Sweden.

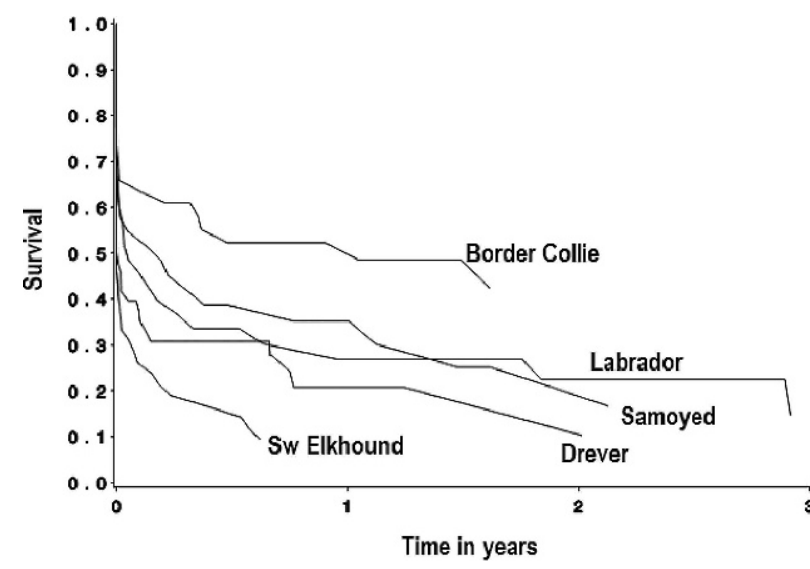

Fig 4. Kaplan-Meier breed-specific survival estimates after 1st insurance claim for DM. Breed-specific survivals are shown for breeds with at least 40 cases. The analysis is based on dogs of 5 10 years of age with life insurance during 1995 to 2004 in Sweden. Dogs insured by Agria Insurance Company, Stockholm, Sweden.

be explained by the fact that the diagnosis of $\mathrm{DM}$ is quite straightforward and not likely to be overlooked in primary care facilities.

One study showed a 1-year survival rate of $64 \%$ for dogs that survived initial stabilization, which is comparable with dogs surviving the initial period in the present study.${ }^{14}$ Approximately $40 \%$ of the dogs in the present study had a death date that coincided with the date of DM claim, probably reflecting a high rate of elective euthanasia at diagnosis. Another reason for dying shortly after diagnosis is diabetic ketoacidosis. Ketoacidosis is reported to occur in $15 \%$ dogs with DM presented to tertiary clinics. ${ }^{27}$ Previous research indicates that $30 \%$ of dogs with ketoacidosis do not survive initial treatment. ${ }^{28}$

In a US study of a hospital record database, it was concluded that Samoyeds, Miniature Schnauzers, and Miniature Poodles were at high risk of DM. ${ }^{5}$ Another survey confirmed these findings and also identified 4 Northern "Spitz" or husky-type breeds (Keeshond, Samoyed, Finnish Spitz, and Siberian Husky) to be among the 12 breeds with high risk of DM. ${ }^{2}$ In the present study, 3 of the 10 breeds with the highest incidence were of Spitz type: Samoyed, Swedish Elkhound, and Swedish Lapphund. Moreover, 3 others of these 10 breeds were Scandinavian hound dogs: Finnish Hound, Hamilton Hound, and Drever. No breed of Scandinavian origin had a lower incidence rate than the mixed breed group. Why Spitz breeds and breeds of Scandinavian origin would be more prone to develop DM than other breeds is not known. Species adapted to cold climate may have altered glucose metabolism that allows them to survive in cold climates and also makes them more susceptible to DM. ${ }^{29}$ Sweden and Finland have among the world's highest incidences of human type $1 \mathrm{DM} \cdot{ }^{15}$ Of the other overrepresented breeds in the present study, the Australian Terrier was previously reported to be predisposed to develop DM. ${ }^{2,3}$

As in other studies, some breeds had a low risk for developing DM (eg, Golden Retriever, Boxer, Papillon, 
Tibetan Spaniel). Furthermore, we found that in Swedish Elkhound, Norwegian Elkhound, Beagle, and Border Collie breeds, females almost exclusively were affected, whereas breeds such as the Labrador Retriever and West Highland White Terrier had equal male/female ratios. These results clearly indicate that some breeds may be prone to types of DM that primarily affect females (eg, progesterone-induced DM). In studies from other countries, these breeds (Beagle, Border Collie, Swedish Elkhound, and Norwegian Elkhound) are not overrepresented, which might be explained by the low number of intact bitches with DM in these studies. The mean age $(95 \% \mathrm{CI})$ at 1 st $\mathrm{DM}$ claim varied significantly with breed, from 7.8 years to 9.3 years. Some breeds have longer life spans than others, which could interfere with the interpretation of the breed-specific means of age of onset. ${ }^{30}$ In the comparison of the 5 breeds that had $>40$ cases, there were significant differences in survival among breeds. In the pairwise comparison, the 2 hunting breeds (Swedish Elkhound and Drever) had lower survival rates than did some of the other breeds, which could be caused by owner-related reluctance to treat hunting dogs with insulin or other breed-related factors related to shorter survival. The differences in sex proportion, age, and survival discussed above indicate that the etiology of DM might vary among breeds and suggests the possibility to map genes involved in the etiology of DM in different breeds.

A study based on medical records from 221 dogs with DM shows that dogs with DM often have concurrent disorders. ${ }^{27}$ The most common concurrent disorders in that study were hormonal disturbances, urinary tract infections, otitis, dermatitis, acute pancreatitis, and neoplasia. Another study also describes acute pancreatitis to be associated with DM. ${ }^{9}$ In the present study, we evaluated endocrine and pancreatic disease episodes as risk factors for DM. In the multivariable analyses, only previous hyperadrenocorticism showed a significantly increased risk for developing DM, which supports the hypothesis that hyperadrenocorticism is a contributing factor for development of DM in dogs and may increase the incidence of DM in breeds susceptible to hyperadrenocorticism. The increased plasma concentration of cortisol in dogs with hyperadrenocorticism increases the plasma glucose concentration and probably also produces an insulin-resistance effect on target cells. None of the other included pancreatic or endocrine diseases showed a significant correlation with DM. In this type of study, there is a risk of underestimating the occurrence of diseases that are difficult to diagnose.

In the multivariable analysis we used a shared frailty effect for breed, corresponding to the effect of a group (random effect) in survival analysis. In a shared frailty model, the frailty effect can be considered to represent the effects of unmeasured predictors that the individuals have in common. ${ }^{31}$ In an early model in which we did not control for breed effect, there were significant effects of earlier episodes of pancreatitis, adrenocortical insufficiency, and hyperadrenocorticism for DM. This effect disappeared (except for hyperadrenocorticism) when we added the breed effect, indicating that breed was a confounder for these diseases. This result emphasizes the importance of controlling for potential breed effects in clinical studies.

\section{Conclusion}

The present study confirms the results of earlier epidemiologic studies regarding the variability in the incidence of DM among different dog breeds. The significant breed-specific, sex, and age differences shown in this study indicate that genetic variation could make breeds more or less susceptible to different types of DM. The results of this study require further investigation, primarily concerning the reasons for the differences in disease characteristics among various breeds, and breedspecific studies on the molecular genetics of DM in dogs are warranted.

\section{Footnotes}

${ }^{a}$ Agria Insurance Company, Stockholm, Sweden

${ }^{\mathrm{b}}$ SAS Institute Inc, Cary, NC

${ }^{\mathrm{c}}$ Stata Corporation, College Station, TX

\section{Acknowledgments}

This study was supported by grants from the Swedish Research Council and the Foundation for Research, Agria Insurance Company. The authors wish to thank Agria Insurance for allowing access to the insurance database. The work was performed at the Department of Clinical Sciences, Uppsala, Sweden.

\section{References}

1. Fracassi F, Pietra M, Boari A, et al. Breed distribution of canine diabetes mellitus in Italy. Vet Res Commun 2004;28(Suppl):339-342.

2. Guptill L, Glickman L, Glickman N. Time trends and risk factors for diabetes mellitus in dogs: Analysis of veterinary medical data base records (1970-1999). Vet J 2003;165:240-247.

3. Davison LJ, Herrtage ME, Catchpole B. Study of 253 dogs in the United Kingdom with diabetes mellitus. Vet Rec 2005; 156:467-471.

4. Krook L, Larsson S, Rooney JR. The interrelationship of diabetes mellitus, obesity, and pyometra in the dog. Am J Vet Res 1960;21:120-127.

5. Hess RS, Kass PH, Ward CR. Breed distribution of dogs with diabetes mellitus admitted to a tertiary care facility. J Am Vet Med Assoc 2000;216:1414-1417.

6. Marmor M, Willeberg P, Glickman LT, et al. Epizootiologic patterns of diabetes mellitus in dogs. Am J Vet Res 1982;43: 465-470.

7. Catchpole B, Ristic JM, Fleeman LM, Davison LJ. Canine diabetes mellitus: Can old dogs teach us new tricks? Diabetologia 2005;48:1948-1956.

8. Imamura T, Koffler M, Helderman JH, et al. Severe diabetes induced in subtotally depancreatized dogs by sustained hyperglycemia. Diabetes 1988;37:600-609. 
9. Cook AK, Breitschwerdt EB, Levine JF, et al. Risk factors associated with acute pancreatitis in dogs: 101 cases (1985-1990). J Am Vet Med Assoc 1993;203:673-679.

10. Hoenig M, Dawe DL. A qualitative assay for beta cell antibodies. Preliminary results in dogs with diabetes mellitus. Vet Immunol Immunopathol 1992;32:195-203.

11. Peterson ME, Altszuler N, Nichols CE. Decreased insulin sensitivity and glucose tolerance in spontaneous canine hyperadrenocorticism. Res Vet Sci 1984;36:177-182.

12. Eigenmann JE, Eigenmann RY, Rijnberk A, et al. Progesterone-controlled growth hormone overproduction and naturally occurring canine diabetes and acromegaly. Acta Endocrinol (Copenh) 1983;104:167-176.

13. Nelson RW. Diabetes Mellitus. In: Ettinger S, Feldman EC, eds. Textbook of Veterinary Internal Medicine, 6th ed. St Louis, MO: Elsevier Saunders; 2005:1563-1591.

14. Doxey DL, Milne EM, Mackenzie CP. Canine diabetes mellitus: A retrospective survey. J Small Anim Pract 1985;26:555-561

15. Karvonen M, Viik-Kajander M, Moltchanova E, et al. Incidence of childhood type 1 diabetes worldwide. Diabetes Mondiale (DiaMond) Project Group. Diabetes Care 2000;23: 1516-1526.

16. Levy-Marchal C, Patterson C, Green A. Variation by age group and seasonality at diagnosis of childhood IDDM in Europe. The EURODIAB ACE Study Group. Diabetologia 1995;38:823-830.

17. Patterson CC, Carson DJ, Hadden DR. Epidemiology of childhood IDDM in Northern Ireland 1989-1994: Low incidence in areas with highest population density and most household crowding. Northern Ireland Diabetes Study Group. Diabetologia 1996;39:1063-1069.

18. Atkins CE, MacDonald MJ. Canine diabetes mellitus has a seasonal incidence: Implications relevant to human diabetes. Diabetes Res 1987;5:83-87.

19. Egenvall A, Hedhammar A, Bonnett BN, Olson P. Survey of the Swedish dog population: Age, sex, breed, location and enrollment in animal insurance. Acta Vet Scand 1999;40:231-240.
20. Egenvall A, Bonnett BN, Olson P, Hedhammar A. Sex, age, breed and distribution of morbidity and mortality in insured dogs in Sweden during 1995 and 1996. Vet Rec 2000;146:519-525.

21. Egenvall A, Bonnett BN, Shoukri M, et al. Age pattern of mortality in eight breeds of insured dogs in Sweden. Prev Vet Med 2000;46:1-14.

22. Swedish Animal Hospital Organisation, Diagnostic Registry for the horse, dog and cat. In: Olson P, Kängström L-E, eds. Taberg, Tabergs tryckeri Stockholm, Sweden: Svenska djursjukhus föreningen; 1993.

23. Allison P. Survival Analysis Using the $\mathrm{SAS}^{\circledR}$ System: A Practical Guide. Cary, NC: SAS Institute Inc; 1995.

24. Egenvall A, Bonnett BN, Olson P, Hedhammar A. Validation of computerized Swedish dog and cat insurance data against veterinary practice records. Prev Vet Med 1998;36: $51-65$.

25. Fleegler FM, Rogers KD, Drash A, et al. Age, sex, and season of onset of juvenile diabetes in different geographic areas. Pediatrics 1979;63:374-379.

26. Warram JH, Krolewski AS. Epidemiology of Diabetes Mellitus. In: Kahn CR, et al., eds. Joslin's Diabetes Mellitus. Philadelphia: Lippincott Williams \& Wilkins; 2005.

27. Hess RS, Saunders HM, Van Winkle TJ, Ward CR. Concurrent disorders in dogs with diabetes mellitus: 221 cases (1993-1998). J Am Vet Med Assoc 2000;217:1166-1173.

28. Hume DZ, Drobatz KJ, Hess RS. Outcome of dogs with diabetic ketoacidosis: 127 dogs (1993-2003). J Vet Intern Med 2006;20:547-555.

29. Moalem S, Storey KB, Percy ME, et al. The sweet thing about type 1 diabetes: A cryoprotective evolutionary adaptation. Med Hypotheses 2005;65:8-16.

30. Egenvall A, Bonnett BN, Hedhammar A, Olson P. Mortality in over 350,000 insured Swedish dogs from 1995-2000. II. Breed-specific age and survival patterns and relative risk for causes of death. Acta Vet Scand 2005;46:121-136.

31. Dohoo I, Martin W, Stryhn H. Modelling survival data. In: Veterinary Epidemilogic Research. Charlottetown, PEI, Canada: AVC Inc; 2003:409-457. 